\title{
Antimicrobial resistance in livestock health: an overview
}

\section{Opinion}

Antimicrobial resistance (AMR) is a global and multispectral problem and indeed, the World Health Organization (WHO) has named AMR as one of the three most important public health threats of the 21 st century. A recent report suggested that AMR is estimated to cause around 300 million premature deaths by 2050 , with a loss of up to $\$ 100$ trillion to the global economy (3.5\% decrease in global GDP). Antibiotics are integral components for maintaining human and animal health and welfare which ultimately contribute to the increasing global demand for safe meat, milk, fish and eggs and other products of animal origin. But, the misuse of antibiotics can result in the development of AMR bacteria. Although the AMR development is a natural phenomena, inappropriate and excessive use of antimicrobials in both human health and in the agricultural sector can speeds up the process. However, India is among the nations with the highest burden of bacterial infections. An estimated 4,10,000 children aged five years or less die from pneumonia in India annually; with pneumonia accounting for almost $25 \%$ of all child deaths. The crude mortality from infectious diseases in India recently 417 per 100,000 persons.

In 2008, about $29 \%$ of isolates of Staphylococcus aureus were methicillin resistant, and by 2014 , this had risen to $47 \%$. The pressure is on India, the world's largest consumer of antibiotics and where carelessness in antibiotic use and environmental antibiotic pollution has been documented. Among the various factors causing the AMR development in pathogens, irrational use of antibiotics in human and agricultural sector particularly in food producing animals is major determinant of AMR development. The association between increased antibiotic use and drug resistance development has been documented in human and veterinary medicines. Quantitative data on antimicrobial use in food animals sectors are also reported regularly by EU, USA, OIE, etc. For instances, comprehensive, integrated surveillance programs operate in the European Union (EU) through European Surveillance of Veterinary Antimicrobial Consumption (ESVAC, 2016) and report on antimicrobial consumption are published annually. In USA, Food and Drug Administration (FDA, 2016) publish its summary report on antimicrobials sold or distributed for use in food-producing animals annually.

The recent report shows that sales and distribution of all and medically important antimicrobials increased one and two percent from 2014 through 2015, respectively. Scientific evidence shows that India is the third largest consumer of antimicrobials (3\%) in food animal's production after China (23\%), the United States (13\%), and Brazil (9\%) during 2010, which is expected to increase about $4 \%$ in India during 2030. This indirect estimation based on animal census has many limitations. In most of the developed countries, data on veterinary antimicrobial consumption were found in the form of overall antimicrobial consumption, consumption by livestock species, and by per population corrected unit (PCU). Besides, consumption by different classes of antimicrobials, various pharmaceutical formulations and routes of administration are also available across the countries. However, in majority of the low or middle income
Volume I Issue 5 - 2017

\author{
Palanisamy Sankar, K Ramya \\ Veterinary and Animal Sciences University, India
}

Correspondence: Palanisamy Sankar,Veterinary College and Research institute, Veterinary and Animal Sciences University, India,Tel 759834359I, Email drpsankarster@gmail..com

Received: November 13, 2017 | Published: December 14, 2017

countries including in India, systematic and reliable data on veterinary antimicrobial consumption are not available and have no regulatory framework for the use of antimicrobials in livestock reared for domestic consumption. Globally, only 42 countries have systematic data on the use of antimicrobials in livestock and recently estimated global livestock sector consumption in 2010 were 63151 tones, which is 0.2 to 0.4 percent of total agricultural consumption.

Global antimicrobial consumption will rise by $67 \%$ by 2030 to 105,596 tons, particularly in the BRICS (Brazil, Russia, India, China and South Africa) countries (doubling).The future consumption of antibiotics in animal sector is expected to increase, particularly in pig and poultry sector. Among the various factors causing the AMR development in pathogens, irrational use of antibiotics in human and agricultural sector particularly in food producing animals is major determinant of AMR development. The association between increased antibiotic use and drug resistance development has been documented in human and veterinary medicines. Therefore, understanding the extent of antibiotic usage and knowledge of AMR among various stakeholders are important for further evidence based action on AMR. Several global human and animal health authorities have initiated the quantification of antimicrobial use or consumption data at regional or national levels as it is an important prerequisite for our understanding of AMR problems. However, there are major data gaps on the use of antimicrobials in livestock globally. Data on the quantity and patterns of antimicrobial use will be essential to evaluate the efficacy of potential policy options. The most controversial use of antimicrobials in livestock is their use as growth promoters.

Antibiotics are very important resource for maintenance of health and productivity of animals which contributes immensely to global food security. On other hand, food of animal origin is potential route of exposure for AMR organisms in food chain. Multidimensional and one health approach is needed with more emphasizes at production and processing level practices in agricultural and animal husbandry sector. Implementing 3Rs (Remove the need for antimicrobials, Reduce the amount of antimicrobials, Restrain and control transmission of resistance through the food chain) is required to control AMR. Regular monitoring and investigation of both antibiotic residues and targeted food borne pathogens in food animals are important for evidence based progress. Restricting antimicrobial use in food animals and decreasing antimicrobial resistance reservoirs in animals could have major public health benefits, even if such benefits are difficult to quantify. 


\section{Acknowledgements}

None.

\section{Conflict of interest}

The authors declare that they have no conflict of interest. 